\title{
Updating of locations during whole-body rotations in patients with hemispatial neglect
}

\author{
JOHN W. PHILBECK \\ George Washington University, Washington, D.C. \\ MARLENE BEHRMANN \\ Carnegie Mellon University, Pittsburgh, Pennsylvania \\ and \\ JACK M. LOOMIS \\ University of California, Santa Barbara, California
}

\begin{abstract}
Posterior parietal cortex lesions have been associated with both hemispatial neglect and spatialupdating deficits. Currently, the relation between these processes remains poorly understood. We tested the ability of parietal patients with neglect to update remembered target locations during passive whole-body rotations. The rotations and manual pointing responses were executed with and without vision. During the rotation, the remembered location stayed on the same side of the body midline or crossed the midline. Parietal patients generally underestimated rotations, as compared with control groups, but updated targets equally well on either side of the body midline, regardless of the amount of updating required. Once parietal patients have localized a target, they can use self-motion information to update its location, even if it passes into the region they typically neglect. This lack of contralesional updating effects contrasts with impairments in eye position updating found in previous work with parietal patients.
\end{abstract}

Many animals have the ability to maintain an awareness of the location of objects that are no longer in view. There is abundant evidence of this, from the skill with which rodents navigate in the dark to the proficient memoryguided saccades and reaches exhibited by monkeys (Colby, Duhamel, \& Goldberg, 1996; Poucet, 1993; Snyder, Batista, \& Andersen, 1997). This ability is present in humans as well, and it can greatly enhance an individual's ability to interact effectively with the world (Loomis, Klatzky, Golledge, \& Philbeck, 1999; Rieser, 1989). It is a common occurrence for objects and locations to be obscured from vision because they pass behind the head or are occluded by other objects or because the lighting conditions change suddenly. Nevertheless, we perceive these objects and their locations as coherent, despite the lack of visual input, and we can continue to act upon them. Being able to update the remembered location of objects

A preliminary report of this work was presented in November, 1999, at the 40th Annual Meeting of the Psychonomic Society in Los Angeles. This research was supported by NIH Grant F32 MH1 1791 and James S. McDonnell Foundation Grant 97-17 to J.W.P. and by NIH Grant RO1 MH54246 to M.B. The authors thank John Jonides and two anonymous reviewers for helpful comments on a previous version of this manuscript. Correspondence concerning this article should be addressed to J. W. Philbeck, Department of Psychology, George Washington University, 2125 G. Street, N.W., Washington, DC 20052 (e-mail: philbeck@ gwu.edu). can mean the difference between navigating effectively back home and remaining lost or between evading a camouflaged predator and becoming its prey.

Despite the behavioral advantages of this form of spatial updating and its common occurrence in everyday life, its underlying brain mechanisms remain poorly understood. One brain region that seems likely to play a role, however, is the posterior parietal cortex (PPC). This region is known to be involved in functions that are critical for encoding and updating the location of nearby targets (Colby \& Goldberg, 1999; Petit, Orssaud, Tzourio, Mazoyer, \& Berthoz, 1997; Stein, 1992). The PPC is thought to monitor certain kinds of body movements-most notably, changes in eye position (Duhamel, Goldberg, Fitzgibbon, Sirigu, \& Grafman, 1992; Heide, Blankenburg, Zimmermann, \& Kompf, 1995) and arm position (Desmurget et al., 1999; DeSouza et al., 2000; Wolpert, Goodbody, \& Husain, 1998). Furthermore, it has been implicated in performing mental transformations of the body in space, a function that may well be related to body and limb position updating (Bonda, Petrides, Frey, \& Evans, 1995; Sirigu et al., 1996; Zacks, Rypma, Gabrieli, Tversky, \& Glover, 1999). Taken together, these results suggest that the PPC's role in integrating the represented locations of objects with updated information about body position may be quite general and extend to encompass information from a variety of muscle groups and sensory modalities. However, the scope of the PPC's role in this regard is very much an open 
issue (Wolpert \& Ghahramani, 2000) and in particular, relatively few studies have investigated its involvement in relatively large-scale body motions.

Locomotion is a particularly attractive domain for studying such questions. It generates a complex assortment of body motions that must be monitored, and when the location of the body changes, so too change the spatial relationships between the observer and objects in the environment. Thus, if the PPC is fundamentally involved in monitoring whole-body motion and using that information to update remembered object locations, one might expect PPC lesions to impair locomotion-related updating. The evidence for this idea has been mixed. Patients with inferior right PPC lesions, at least, perform quite normally in walking tasks that require nonvisual self-motion updating along linear trajectories (Philbeck, Behrmann, Black, \& Ebert, 2000; see also Bisiach, Pattini, Rusconi, Ricci, \& Bernardini, 1997; Pizzamiglio, Iaria, Berthoz, Galati, \& Guariglia, in press). By contrast, some PPC patients have been shown to significantly underestimate passive whole-body rotations (Farrell \& Robertson, 2000; Tropper, Jones, Bloomberg, \& Fadlallah, 1991). The interpretation of the rotational updating results, however, is complicated by the fact that the inferior PPC borders a region in the posterior temporal lobe that appears to be specialized for processing vestibular information (Friberg, Olsen, Roland, Paulson, \& Lassen, 1985; Lobel, Kleine, Bihan, Leroy-Willig, \& Berthoz, 1998; Vitte et al., 1996). The proximity of this region to the PPC means that brain injuries involving the PPC may also impinge upon the vestibular cortex. After testing a group of PPC-injured patients and a separate group of patients with vestibular cortical lesions, Israël and colleagues (Israël, Rivaud, Gaymard, Berthoz, \& PierrotDeseilligny, 1995) concluded that the PPC itself is not crucial for processing passive rotations. Thus, previous reports of PPC-related deficits on tasks involving wholebody rotations may be at least partially due to vestibular cortical injury.

None of the previous studies, however, attempted to distinguish self-motion updating from updating of remembered target locations. Whether or not there is reduced sensitivity to vestibular stimulation as a result of brain injury, PPC-injured individuals may be impaired at making use of their residual vestibular sensitivity to update the location of remembered targets. If self-motion updating and target updating are behaviorally dissociable, one might predict that, for a given magnitude of perceived rotation, some target locations would be updated better than others. One of the hallmarks of PPC damage potentially provides a means of eliciting this dissociation. PPC damage is often associated with a collection of representational and attentional deficits known as hemispatial (or unilateral) neglect. Neglect is typically manifested as impairments in detecting or acknowledging events in a contralesional region of space (Bisiach \& Vallar, 2001; Vallar, 1998). Given the lateralized representational and/or attentional deficits associated with neglect, one might expect neglect patients to exhibit deficits in updating remembered object locations when body movements cause the object trajectory to pass into the neglected region.

Significantly, PPC-injured individuals show exactly this kind of impairment when saccadic eye movements supply the stimulus to updating, instead of vestibular stimulation (Duhamel et al., 1992; Heide et al., 1995). In the double-step saccade paradigm, observers attempt to sequentially foveate two rapidly flashed visual targets; this requires updating the remembered location of the second target on the basis of changes in the eye position signal (efference copy). PPC patients are impaired at updating ipsilesional targets only when the first saccade makes their eyes point into the contralesional hemifield; these deficits, furthermore, are correlated with the degree of hemispatial neglect. Given the multiplicity of sensory inputs known to converge upon the primate PPC (Andersen, Snyder, Bradley, \& Xing, 1997), this structure's role in updating the remembered location of targets may well incorporate self-motion signals from a variety of sensory systems, including the vestibular apparatus.

To shed light on this possibility, we tested the ability of a group of neglect patients to update remembered target locations during passive whole-body rotations. We presented a visual target to the left or right of the egocentric body midline (contra- and ipsilesional for the brain-injured patients). Vision was then occluded, and the observer underwent a passive whole-body rotation about an earth-vertical axis. During the rotation, the remembered location of the target either remained on the same side of the body midline or crossed over to the opposite side. The task was to use a manual pointer to indicate the target location after the rotation. Thus, in order to point accurately, the participants not only had to monitor the vestibular signals generated by the rotation, but also had to use them to update the remembered target location. By choosing certain combinations of rotation magnitudes and initial target locations, we created stimulus conditions that required different amounts of target updating in each hemispace. We predicted that, for the neglect patients, pointing errors would be associated most strongly with the amount of updating in the contralesional hemispace, rather than with the direction of the body rotation.

Methodologically, passive rotations are advantageous because they restrict the sensory input available for target updating primarily to those signals arising from the vestibular apparatus. In principle, self-generated (active) rotations could also be used, if one assumes that the likelihood of observing contralesional target updating deficits does not depend on the sensory modality that signals the rotation. On this assumption, active rotations should only enhance perception of the body rotation (by virtue of the additional efference signals and proprioception contributed by movement of the arms and/or legs), without differentially affecting the updating processes for targets within the contralesional hemispace. A potential 
drawback of active rotations, however, is that if the participants use their arms or legs to produce the rotation, they might count the number of arm or leg movements made during the rotation. They might then use this information to perform the task without truly sensing the rotation or updating the target location. Passive rotations avoid this pitfall.

There are several potential sources of pointing error in this paradigm. At the very least, the following processes are involved: (1) visual localization/encoding of the target location, (2) perceptuomotorcalibration, (3) localization of the egocentric body midline, (4) perceptual registration of the body rotation, and (5) updating of the remembered target location, on the basis of the perceived body rotation. Target localization and perceptuomotor calibration (1 and 2) are especially important to check in PPC patients, given that parietal lesions are associated with a decreased probability not only of detecting targets on the contralesional side, but also of directing motor behaviors toward that side (Behrmann, Ghiselli-Crippa, \& Di Matteo, 2002; Karnath \& Perenin, 1998). With regard to (3) above, wholebody rotations may influence the subjective midline localization, particularly in individuals, such as parietal patients, who have lateralized attention deficits. Consistent with this view, vestibular stimulation has been found to ameliorate neglect symptoms, suggesting that a midline shift may have occurred (Bisiach \& Vallar, 2001; Rode et al., 1992; Rode \& Perenin, 1994). If the subjective direction of straight ahead changes after a whole-body rotation, one might expect concomitant changes in other directional judgments. In the following experiment, we measured observers' ability to localize visual targets and point to them by memory (points 1 and 2, above), as well as their ability to localize their body midline after wholebody rotations (3). With these data in hand, we then measured perception of rotations (4) and target updating (5) in a series of experimental trials that involved pointing to a remembered target after a whole-body rotation.
METHOD

\section{Participants}

Three groups of observers consented to participate in this study. (1) A healthy control group ( $n=10,5$ women and 5 men; mean age, 67.8 years $^{1}$ ) had no history of neurological disease. (2) The braininjured control group ( $n=5,1$ woman and 4 men; mean age, 42.9 years) had brain injuries of mixed etiologies that spared the right-hemisphere posterior parietal lobe. Two of these patients had right-hemisphere occipito-temporal lesions and were visually agnosic; 2 others had circumscribed left occipital lesions and demonstrated pure alexia. The 5 th had undergone a right frontotemporal lobectomy. These patients have been described in more detail elsewhere (Behrmann, Nelson, \& Sekuler, 1998; Mapelli \& Behrmann, 1997; Philbeck et al., 2000; Vecera \& Behrmann, 1997). Two of these observers had visual field defects that extended from about $5^{\circ}$ right of the fovea outwards to the far right periphery, and a $3 \mathrm{rd}$ had an upper visual field defect extending upwards from about $10^{\circ}$ of the fovea. However, all of these patients could localize the stimuli without difficulty by making eye and/or head movements, and 4 out of the 5 tested within normal limits on the Behavioral Inattention Test (BIT; Wilson, Cockburn, \& Halligan, 1987), a standard test designed to measure hemispatial neglect (see Table 1). The fifth patient was not tested, because of his severe impairments in visual perception. These deficits would cause him to perform poorly on standard neglect tests, but the deficits would not be differentiated by the side of stimulus presentation (left or right of the midline). His perception was adequate, however, for localizing a single illuminated target. (3) Patients in the parietal group $(n=6,1$ woman and 5 men; mean age, 63.6 years) were selected for inclusion by the presence of a cerebral vascular accident or some other injury that resulted in damage to the parietal lobe. None of these patients had visual field defects that would prevent them from seeing the stimuli. Three of these patients have been described in more detail elsewhere (Philbeck et al., 2000). A 4th patient had a right-hemisphere cerebral vascular accident (CVA) that damaged a fairly large cortical area, involving the right temporoparietal and posterior frontal regions. A 5th patient had a relatively complex etiology, involving a right parieto-temporal CVA and a right frontoparietal temporal craniotomy. These injuries were followed by insertion of a ventriculoperitoneal shunt from the right PPC region. In addition to the damage caused by the shunt surgery itself, chronic infection and edema developed around the shunt, eventually necessitating its removal. A left parietal ventriculoperitoneal shunt was subsequently

Table 1

Demographic and Neglect Details for Patients in the Parietal and Brain-Injured Control Groups

\begin{tabular}{lllllrr}
\hline Patient & Group & Sex & Age & Hand* & Time Test $\dagger$ & Neglect Score \\
\hline J.B. & parietal & male & 68 & right & 53 & $\mathbf{1 0 3}$ \\
R.B. & parietal & male & 66 & right & 67 & $\mathbf{1 0 8}$ \\
L.D. & parietal & male & 76 & right & 11 & $\mathbf{8 6}$ \\
T.G. & parietal & male & 46 & left & 15 & $\mathbf{1 1 4}$ \\
J.S. & parietal & male & 71 & right & 42 & $\mathbf{1 0 5}$ \\
C.V. & parietal & female & 52 & right & 44 & $\mathbf{1 2 3}$ \\
J.W. & control & male & 41 & left & 37 & 143 \\
E.L. & control & female & 49 & right & 24 & 143 \\
D.K. & control & male & 68 & right & 68 & 141 \\
J.S. & control & male & 26 & right & 69 & 146 \\
R.D. & control & male & 24 & right & 31 & \\
\hline
\end{tabular}

*Preferred hand. †Time of testing, postonset (months). $\quad$ ॠMaximum score $=146$; boldface indicates belownormal performance. $\quad * *$ Not measured; J.W. is likely to perform poorly on standard neglect tests because of his perceptual impairments, but this poor performance would not be differentiated by side (left or right). 
inserted. Thus, this patient had bilateral parietal damage, but because she did show left-sided neglect, we considered left-sided targets to be contralesional for her. The 6th patient in this group had a history of astrocytoma that was associated with damage in the medial left parietal region. He had also undergone a left temporo-parietal craniotomy. In contrast to the other patients in this group, he demonstrated right-sided neglect. All 6 of these patients scored below normal on the BIT. Table 1 presents the demographic details and lesion information of the two patient groups, as well as neglect scores for the patients. Figure 1 shows neuroimaging data for 5 of the 6 patients in the parietal group; scans for the 6th patient were not available for publication.

\section{Apparatus}

The experiment took place in a well-lit room. The apparatus, the room context, and all possible target locations were therefore visible when a particular target was specified during the experiment. This provided multiple visual cues to reinforce the target location when it was specified. The participant sat in a swivel chair, with his or her feet placed on the chair's footrest. A manual pointing device, used to register directional responses, was mounted horizontally on the chair in the observer's median sagittal plane, at about the level of the abdomen (see Figure 2). The pointer's axis of rotation was offset $23 \mathrm{~cm}$ from that of the chair. The pointer itself was a thin rod extending $16 \mathrm{~cm}$ from its axis of rotation. A printed polar scale graduated to the nearest degree lay just under the rod. The experimenter recorded pointing responses by reading off the value on the scale indicated by the rod's pointing direction.

The chair was secured to the floor in the middle of a table, $1.52 \mathrm{~m}$ square and $76 \mathrm{~cm}$ tall. The chair protruded through a circular aperture in the table, $76 \mathrm{~cm}$ in diameter (a removable panel in the table provided the participant access to the chair). At the beginning of each trial, the participant was always oriented toward the same designated origin - a visible reference point on the table lying in the middle of one of its sides and $76 \mathrm{~cm}$ away from the chair's rotation axis. The participant's orientation was set by aligning markers on the arms and back of the chair so that they were in register with corresponding positioning marks on the table. Four electric lamps $(12 \mathrm{~V}, 80 \mathrm{~mA})$ were mounted on the table at a radius of $76 \mathrm{~cm}$ from the rotation axis of the chair and at eccentricities of $25^{\circ}$ and $65^{\circ}$ on the left and right of the origin. ${ }^{2}$ On certain trials of the experiment, one of these lamps was illuminated, flashing at about $7 \mathrm{~Hz}$ for $2 \mathrm{sec}$.

\section{Design}

The task on each trial was to use the manual pointing apparatus to indicate the direction to a specified target. There were three main trial types. The trials were blocked by condition, and the running order was fully randomized within each block.

Perceptuomotor performance trials. On these trials, we measured the observers' ability to point to each of the four target positions, as well as the origin reference point lying straight ahead, without a passive rotation imposed before the response. This allowed us to estimate the ability of each observer to localize the targets, remember their locations, and point to them with the pointing apparatus. The observers pointed to each target three times in random order, first with vision during the response and then in a second block with eyes covered during the response.

Midline shift trials. On these trials, the observers pointed straight ahead after passive rotations of $75^{\circ}$ and $125^{\circ}$ to either side (three times per condition). This allowed us to estimate the degree to which passive rotations influenced the localization of the body midline. These trials were conducted without vision during the response.

Experimental trials. Our primary concern was to look for impairments related to the amount of target updating that was required within the PPC group's contralesional hemispace. By using various com- binations of body rotations and initial target bearings (see Table 2), the design ensured that the target location sometimes remained on the same side of the body midline during the rotation and sometimes crossed over, thereby requiring a range of amounts of target updating in each hemispace. In one block of trials, the target was the origin-a landmark visible at the edge of the table in the observer's egocentric body midline at the beginning of the trial. In another block, the target was a flashing light at $25^{\circ}$ or $65^{\circ}$ to the left or right relative to the observer's starting orientation. There were 10 possible magnitudes of passive rotation $\left(25^{\circ}-125^{\circ}\right.$ in $25^{\circ}$ increments on the left and right sides). One arbitrary constraint placed on the sampling was that the direction of the passive rotation was always toward the target's initial position. Out of a large number of possible target-rotation pairings, we selected 14 combinations for rightward and leftward rotations, yielding a total of 28. Each of these combinations was generally measured six times apiece (in some cases, as few as three times apiece, in the event of observer fatigue). Responses in these experimental trials were collected without vision during the response. Prior to collecting the no-vision responses, however, an abbreviated set of responses was collected under visual control. These practice trials allowed us to verify that the observers understood the task before attempting to perform them without vision.

\section{Procedure}

The observers were first alerted to the fact that the pointer's center of rotation was offset from that of the chair. Using a point on the table $90^{\circ}$ to the left of the chair's rotation axis as an example, the experimenter demonstrated that an accurate pointing response would require the pointer to be set more than $90^{\circ}$ to the left because of the offset. The observers were instructed to use the right hand to set the pointer so that an imaginary line passing through the pointer rod would connect directly with the physical location of the designated target. One left-handed patient in the parietal group had some rightsided hemiparesis as a result of his left-hemisphere damage, so he manipulated the pointer by using his left hand. For straight ahead judgments, the observers were instructed to imagine a line extending from their trunk pointing straight ahead and to set the pointer to be collinear with this imaginary line. After every pointing response in the experiment, the experimenter returned the pointer to a starting position that pointed directly toward the observer's abdomen. This ensured that all the responses required some amount of motor behavior to set the pointer.

On trials in which the response was conducted without vision, observers began each trial oriented toward the reference origin with the blindfold raised. During a short delay, they either viewed one of the target lights as it was illuminated or viewed the reference origin to firmly establish the starting orientation. After viewing the target, they then donned the blindfold and made the pointing response either immediately (perceptuomotor performance trials) or after the experimenter had executed a passive rotation (all other trials). The delay imposed by passive rotations was less than $2 \mathrm{sec}$. On rotation trials, the observer wore hearing protectors (overall noise reduction rating, $20 \mathrm{~dB}$ ) to minimize potential auditory cues as to his or her orientation in the room. No feedback about error was given, except on those trials conducted under visual guidance. Head motion was unconstrained during the initial localization of the target, as well as during the stimulus rotation and pointing responses. In practice, the observers tended to rotate the head slightly toward the target during the stimulus presentation and then return it to a position aligned with the trunk before covering the eyes. They then tended to keep the head in this position during the rotation and response phases of the trial.

The experimenter stood directly behind the observer and executed the rotations by manually turning the chair. Before beginning the experiment, the experimenter had administered over 1,000 rotations in a pilot experiment and had become adept at executing the 


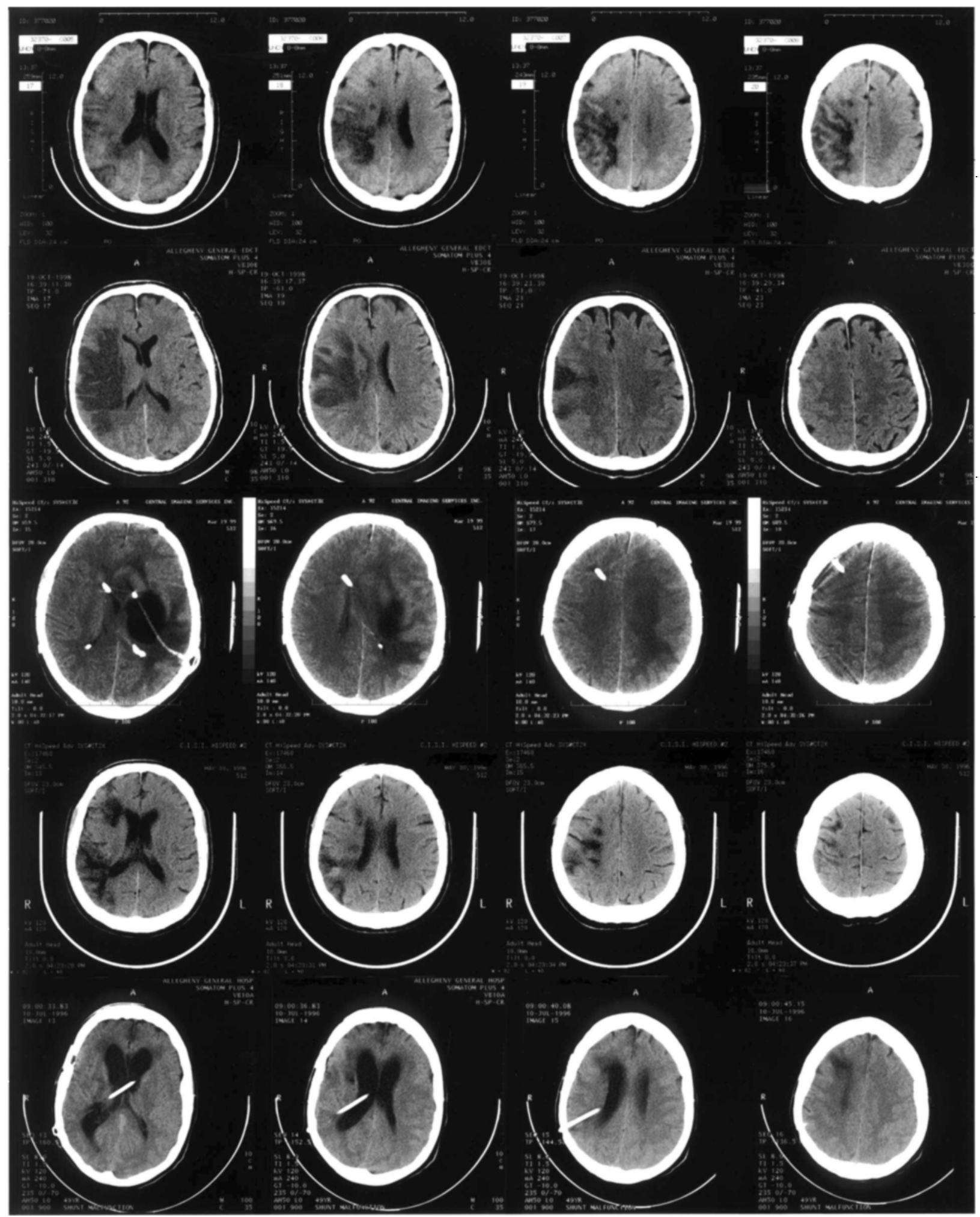

Figure 1. Neuroimaging data (by computerized tomography) for 5 of the 6 patients in the parietal group. Images of patient R.B. were not available for publication. Each row of images is from the same patient, and the sequence of patients follows the same order at that in Table 1. From top to bottom: J.B., L.D., T.G., J.S., and C.V. Radiological coordinates are used, with the right hemisphere appearing on the left side of the images and the anterior of the brain appearing on the top. 


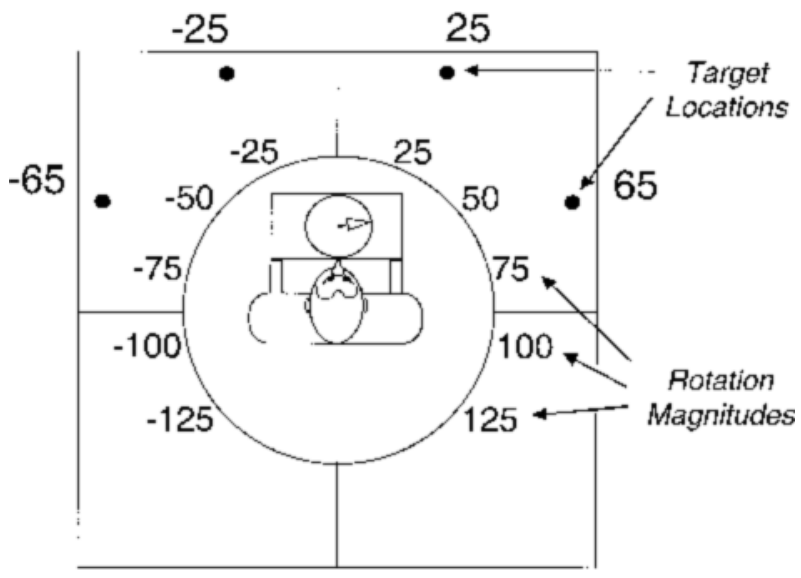

Figure 2. Schematic diagram of the experimental apparatus, seen from overhead. The observer sits in a swivel chair and uses a pointing device to indicate the direction to specified targets. The pointing device is mounted on the chair and rotates with it. The chair itself sits in the middle of a circular aperture cut into a square table. Target lamps are placed on the table surface at $\mathbf{2 5}^{\circ}$ and $65^{\circ}$ on either side of the observer's starting orientation.

rotations, using a stereotyped velocity profile. In particular, he attempted to impose a bell-shaped velocity profile, with rapid, monotonic accelerating and decelerating phases and a minimum of jerky starts and stops. Bell-shaped velocity profiles are preferred when studying vestibular function, because they are thought to approximate those imposed by natural head movements (Blouin, Gauthier, \& Vercher, 1995; Uemura, Arai, \& Shimazaki, 1980). Typical velocity profiles were recorded with an infrared video tracking system (Optotrak, Waterloo) as the experimenter rotated a volunteer, using the same stereotyped motions as in the actual experiment. The peak angular velocity varied from about $40^{\circ}$ to $90^{\circ}$ per second, depending on the rotation magnitude. The marks on the table used to position the chair were flanked by scale markers graduated to the nearest degree; the accuracy of each rotation relative to the nominal value was recorded on each trial by referring to the scale markers. Subsequent analyses of pointing errors reflected the actual stimulus rotations.

\section{Data Analysis}

Prior to the analysis, the data were averaged across repetitions within observers, and outliers more than four standard deviations from the mean were removed. This resulted in the removal of about $2 \%$ of the data. To facilitate comparison of the pointing responses with the target directions and rotation magnitudes, we transformed the responses to the corresponding values that would be produced if the pointer axis were coincident with the chair's rotation axis. For the patients, we coded the direction of target locations, rotations, and pointing responses relative to the hemisphere of the brain injury (i.e., ipsi- or contralesional). The majority of the patients had righthemisphere lesions, so a target was typically considered contralesional when it was situated to the observer's left.

Target bearings and body rotations were defined relative to the observer's initial orientation of $0^{\circ}$, with a maximum possible value of $180^{\circ}$. Leftward targets and rotations were scored as negative. Pointing responses were defined relative to the observer's orientation at the time of responding, with $0^{\circ}$ corresponding to straight ahead and negative and positive values being to the left and right of straight ahead, respectively. For each statistical test mentioned below, we conducted analyses on both signed and absolute pointing errors, but unless otherwise indicated, we will present only the signed error data because the two analyses produced very similar results. Signed errors were calculated so that negative errors always indicated that the pointing response was smaller than required. For example, if the nominally correct pointing response was $100^{\circ}$ to the right, an actual pointing response of $90^{\circ}$ to the right would yield a pointing error of $-10^{\circ}$. Similarly, a nominal leftward response of $-100^{\circ}$ with an actual response of $-90^{\circ}$ would also yield an error of $-10^{\circ}$.

Analysis of experimental trials. Our primary goal in this study was to examine the experimental conditions for evidence of target updating deficits within the PPC group's contralesional hemispace. To do this, we performed two types of analyses of variance (ANOVAs)

Table 2

Stimulus Combinations Used in the Experiment

\begin{tabular}{lcccc}
\hline \multicolumn{1}{c}{ Condition } & Vision?* & Rotation? & Target $(\mathrm{deg}) \dagger$ & Rotation (deg) \\
\hline Perceptuomotor, with vision & yes & no & $0,25,65$ & N/A \\
Perceptuomotor, no vision & no & no & $0,25,65$ & N/A \\
Midline shift & no & yes & $0 \S$ & 75,125 \\
Experimental & no\# & yes & 0 & 125 \\
& & & 0 & 100 \\
& & 0 & 75 \\
& & 0 & 50 \\
& & 0 & 25 \\
& & 25 & 100 \\
& & 25 & 75 \\
& & 25 & 50 \\
& & 25 & 25 \\
& & 65 & 125 \\
& & 65 & 100 \\
& & 65 & 75 \\
& & 65 & 50 \\
\hline
\end{tabular}

*Indicates presence or absence of vision during response execution. †Initial target bearing; nonzero values were presented on the left and right sides of the egocentric body midline. $\$$ Magnitude of the whole-body rotation about an earth-vertical axis; rotation directions were toward the left and right sides of the egocentric body midline. \$Straight ahead, relative to participant's orientation after the rotation. \#Several experimental trials were conducted with vision during the response to familiarize participants with the procedure. 

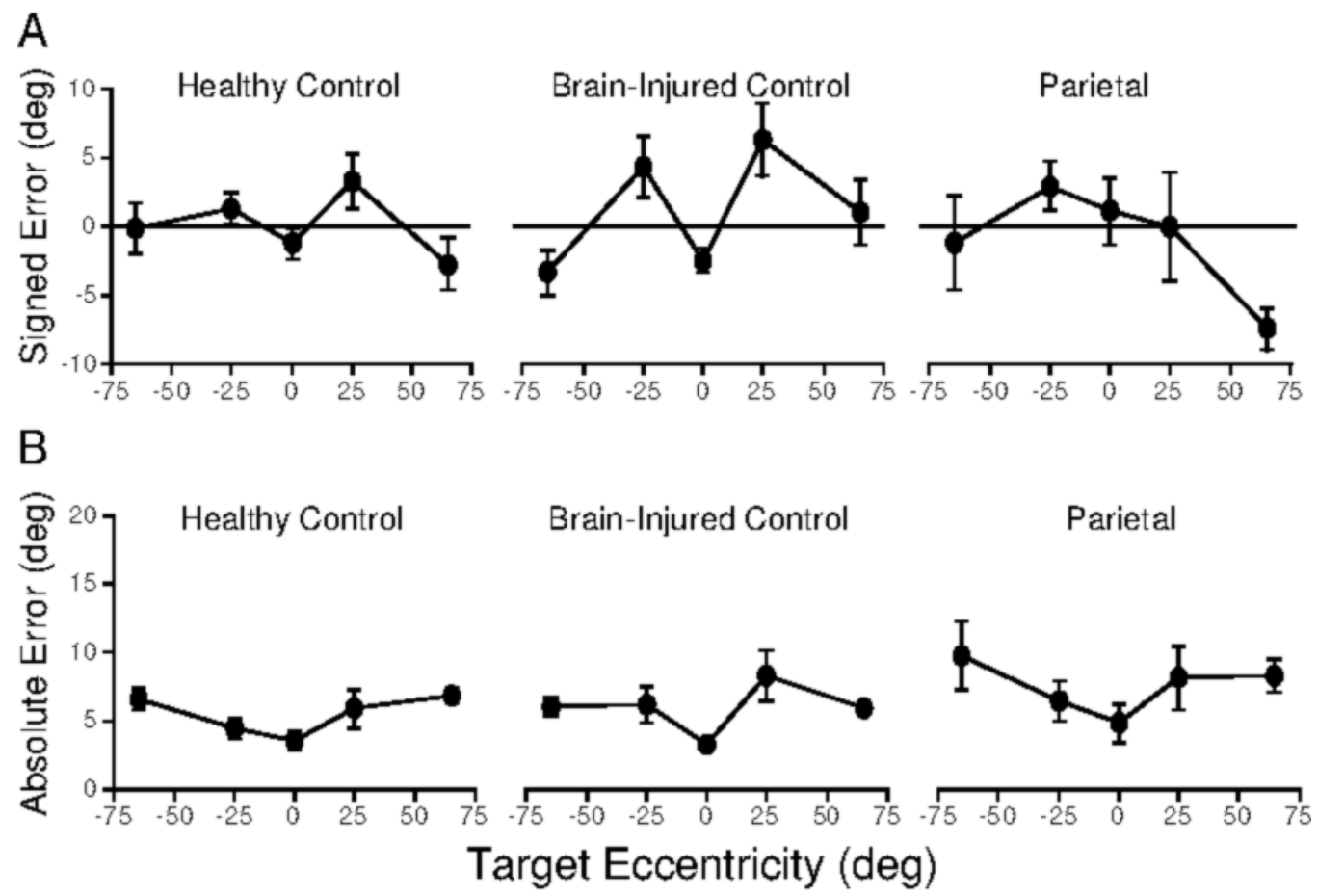

Figure 3. Mean pointing error on the perceptuomotor performance trials for the three groups. Pointing error is shown as a function of the five possible target eccentricities. Pointing responses were made without vision, and no whole-body rotations were imposed before responding. Error bars denote \pm 1 standard error of the mean. (A) Mean signed pointing error; negative values signify undershoots of the required value. (B) Mean absolute pointing error.

on the pointing error data from the experimental trials. (1) The initial target bearing test analyzed the data according to experimentermanipulated variables, such as initial target bearing (origin vs. $25^{\circ}$ vs. $65^{\circ}$ ), rotation direction, and rotation magnitude. (2) The target updating test divided the experimental data into three categories based on the amount of target updating that was required within the left versus right hemispace. These categories could not be determined and manipulated a priori, because the amount of updating in each hemispace depended critically on the perceived magnitude of the whole-body rotations. Even if a target physically crossed the midline during a body rotation, underregistration of the rotation might mean that the individual perceived the target to remain in the same hemispace throughout the trial. To deal with this, we categorized experimental trials as crossover or no-crossover according to whether or not the participant's pointing response (and not the experimenterdefined initial target bearing) indicated that the target crossed the body midline during the body rotation. A third category in the target updating analysis consisted of the point-to-origin trials.

Because the remembered target location remained on the same side of the body midline throughout no-crossover trials, we assumed that either $100 \%$ or $0 \%$ of the required target updating was within the contralesional hemispace, depending on the rotation direction. We predicted that parietal patients would perform nocrossover trials involving contralesional rotations more poorly because $100 \%$ of the updating occurred in their contralesional hemispace. Crossover trials entailed some intermediate amount of updating within the contralesional hemispace, for both rightward and leftward rotations. On the basis of the double-step saccade literature (Duhamel et al., 1992; Heide et al., 1995), we predicted that parietal patients would perform more poorly on crossover trials that in- volved a contralesional target that crossed into the ipsilesional hemispace during a body rotation toward the contralesional side. In terms of updating, point-to-origin trials were similar to the nocrossover conditions in that the majority of target updating was either inside or outside the contralesional hemispace, depending on the rotation direction. On point-to-origin trials, however, updating in the contralesional hemispace was produced by an ipsilesional rotation. Thus, we predicted that parietal patients would perform more poorly on point-to-origin trials involving turns toward the ipsilesional side. The rotation direction comparison was crucial for assessing the role of the PPC in updating target locations; if the PPC plays a critical role in target location updating, one would expect the deficits exhibited by the PPC patients to be associated with the amount of updating in the contralesional hemispace, rather than with the direction of body rotation.

\section{RESULTS}

The primary findings, based on analyses of the experimental trials, indicated that (1) the PPC group tended to underestimate the magnitude of passive, whole-body rotations to a greater degree than did the control groups and (2) contrary to our predictions, their pointing errors did not depend on the amount of required target updating within the contralesional hemispace. Before turning to those data, however, the results of the perceptuomotor performance trials, midline shift trials, and visually guided practice trials will be presented. The multiplicity of con- 


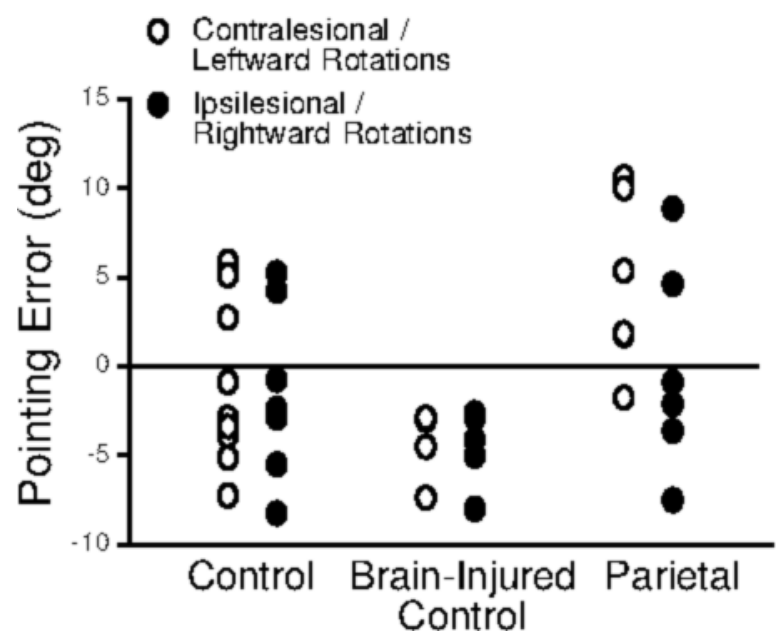

Figure 4. Signed pointing errors for straight ahead judgments on the midline shift trials for the three groups. Each symbol represents the mean error for one individual. Judgments made after contralesional and ipsilesional rotations are shown. Contralesional and ipsilesional rotations correspond to leftward and rightward rotations, respectively, for the healthy control group. Negative values signify undershoots of the required value.

trol conditions dictated that a relatively large number of statistical tests were required; most of these tests were descriptive in nature and were not direct tests of our hypotheses. Rather than apply a correction for multiple comparisons, we will present the mean squared errors and exact $p$ values to give an indication of effect size.

\section{Perceptuomotor Performance Trials}

We first compared visually guided responses on perceptuomotor performance trials with responses made without vision. Analyses of the signed pointing error showed that all three groups performed very similarly $\left[F(2,18)=0.647, M S_{\mathrm{e}}=38.57, p=.536\right]$, with the pointing error for each group being within $2^{\circ}$ of accurate on average. The no-vision responses did not differ from those performed under visual control $\left[F(1,18)=1.56, M S_{\mathrm{e}}=\right.$ $56.71, p=.228]$, but there was a main effect of target location $\left[F(4,72)=8.376, M S_{\mathrm{e}}=239.14, p=.001\right]$; mean signed errors for the $25^{\circ}$ targets were $3^{\circ}$, with the $-65^{\circ}$ and $0^{\circ}$ targets yielding virtually no systematic error and the $+65^{\circ}$ target being undershot by $2.5^{\circ}$. However, there were no interactions of group with the availability of vision during the response $\left[F(2,18)=0.083, M S_{\mathrm{e}}=3.00, p=\right.$ $.921]$ or with target location $\left[F(8,72)=1.451, M S_{\mathrm{e}}=\right.$ $41.417, p=.191]$. For simplicity, Figure 3 presents the data from the no-vision responses only. The group similarities indicate that the patient groups, despite their brain injuries, were able to localize the targets well; furthermore, they could point to these targets with normal accuracy when no whole-body rotations were involved. The good performance of the neglect patients is especially noteworthy. In this regard, it is significant that the patients were given sufficient time to explore both sides of the workspace. In addition, the testing environment was visually well structured, the target stimuli flashed rapidly to draw attention, and the patients were given verbal cues to help them locate the stimuli, if necessary. One account of hemispatial neglect holds that the spatial representation of the left hemispace is compressed in left neglect (Karnath \& Ferber, 1999); if such was true of our patients, the multiplicity of spatial cues apparently had the desired effect of enhancing target localization.

Analyses of the absolute pointing errors were similar, except that there was a small group effect $[F(2,18)=$ $4.185, M S_{\mathrm{e}}=86.934, p=.0322$ ], with the parietal group being only slightly less accurate than the healthy and brain-injured control groups (mean error, $6.2^{\circ}$ vs. $4.2^{\circ}$ and $5.4^{\circ}$, respectively, for the two control groups). In general, responses made with vision were more accurate than those made without vision $\left[F(1,18)=25.192, M S_{\mathrm{e}}=\right.$ $209.973, p=.0001]$, with mean errors of $3.9^{\circ}$ versus $6.2^{\circ}$,

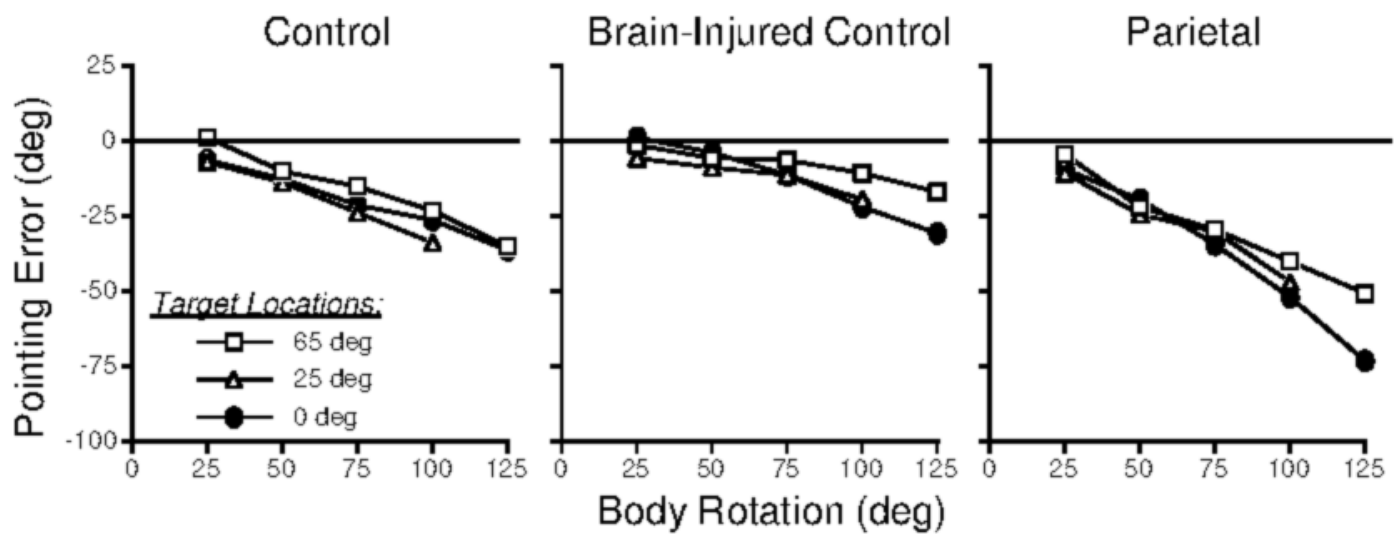

Figure 5. Mean signed pointing errors on the experimental trials for the three groups. Responses made in the context of a target at the origin, $\mathbf{2 5}^{\circ}$ and $6^{\circ}$, are shown. The data have been collapsed across trials involving contralesional and ipsilesional whole-body rotations (leftward and rightward rotations, respectively, for the healthy control group). Negative values signify undershoots of the required value. The $0^{\circ}$ data constitute the point-toorigin trials, whereas the $25^{\circ}$ and $65^{\circ}$ data include a mixture of crossover and no-crossover trials. 
respectively. There was again an effect of target location $\left[F(4,72)=11.404, M S_{\mathrm{e}}=101.906, p=.0001\right]$, so that the mean absolute pointing error tended to increase with target eccentricity. This effect did not interact with group, however $\left[F(8,72)=1.252, M S_{\mathrm{e}}=11.192, p=.282\right]$, confirming that the parietal group pointed in a manner similar to the control groups (see Figure 3B).

The pointing responses on the no-vision trials of this analysis were collected immediately after vision was obscured. In conditions involving whole-body rotation, however, a short delay was interposed between vision of the target and execution of the response, owing to the time required for the rotation. Thus, as compared with trials involving rotation, errors in the perceptuomotor performance trials might be systematically smaller if short delays, by themselves, have a detrimental effect on pointing accuracy. To address this, we tested a subset of the observers (10 healthy controls and 5 of the 6 parietal patients) on perceptuomotor performance trials in which a longer (5-sec) delay was interposed between vision of the targets and response execution-a bit longer than the delay caused by the largest rotation magnitude. We then compared the delay versus no-delay signed pointing errors. The two groups performed similarly overall $[F(1,13)=$ $\left.1.145, M S_{\mathrm{e}}=136.87, p=.304\right]$. There was a small effect of delay $\left[F(1,13)=13.208, M S_{\mathrm{e}}=72.88, p<.003\right]$, with the mean error being only $1.04^{\circ}$ and $-0.18^{\circ}$ for the nodelay and delay conditions, respectively. The delay effect was undifferentiated by group $\left[F(1,13)=3.446, M S_{\mathrm{e}}=\right.$ $19.02, p=.086]$. As in the previous (no-delay) perceptuomotor performance analysis, there was again a main effect of target $\left[F(4,52)=5.913, M S_{\mathrm{e}}=232.79, p=.0005\right]$. In the current analysis, there was also a group $\times$ target interaction $\left[F(4,52)=5.43, M S_{\mathrm{e}}=213.81, p=.001\right]$. The parietal group undershot the $+65^{\circ}$ target by $10^{\circ}$ on average, whereas all other mean pointing errors for both groups were within $4^{\circ}$ of accuracy. Undershooting of the $+65^{\circ}$ target in the parietal group is observable to some extent even in the no-delay trials (see Figure 3A); its cause is unknown, but we take this effect into account in the recalibrated experimental trial analysis below.

\section{Midline Shift Trials}

Interestingly, there was a main effect of group in this analysis $\left[F(2,18)=3.932, M S_{\mathrm{e}}=135.546, p=.0383\right]$, which pairwise planned contrasts revealed was due to a difference between the parietal and the brain-injured control groups [healthy vs. brain-injured control, $F=2.41$, $M S_{\mathrm{e}}=1,058.05, p=.138$; parietal vs. brain-injured control, $F=12.45, M S_{\mathrm{e}}=5,471.85, p=.0024$; parietal vs. healthy control, $\left.F=6.21, M S_{\mathrm{e}}=2,728.91, p=.0227\right]$. The group effect, in turn, depended on whether the body rotation was toward the left or the right side of the observer's midline $\left[F(2,18)=5.871, M S_{\mathrm{e}}=27.459, p=.0109\right]$. Although the two control groups were not affected by the direction of the body rotation, the parietal patients accurately pointed straight ahead after ipsilesional (typically, rightward) rotations but pointed an average of $4.6^{\circ}$ right of straight ahead after contralesional (leftward) rotations (see Figure 4). This is consistent with the notion that wholebody rotations toward the contralesional hemispace shifted the perceived direction of straight ahead slightly into the ipsilesional hemispace for the parietal patients (Karnath, 1994).

\section{Visually Guided Practice Trials}

We conducted ANOVAs on the pointing errors from the visually guided practice trials for the experimental conditions. All the participants completed these practice trials, except for 1 member of the healthy control group. The groups generally performed quite similarly when pointing to origin $\left[F(2,17)=0.144, M S_{\mathrm{e}}=5.48, p=.867\right]$ and nonorigin $\left[F(2,17)=0.084, M S_{\mathrm{e}}=0.772, p=.92\right]$ targets. Pointing errors averaged $-1.4^{\circ}$ and $-3.5^{\circ}$ for the origin and nonorigin targets, respectively. Although there were no main effects involving the direction of rotation, there was a group $\times$ direction interaction on the practice trials involving nonorigin targets $\left[F(2,17)=5.31, M S_{\mathrm{e}}=99.02, p<\right.$ $.016]$. On these trials, there was a slight pointing bias $(M=$ $\left.4.6^{\circ}\right)$ in the brain-injured control group toward the ipsilesional side. Taking the two types of practice trials together, however, there was a high degree of accuracy, and the lack of group effects verified that the observers understood that they were to point to the target's location as it would be situated after the passive rotation.

\section{Experimental Conditions}

With the control data in hand, we now turn to the experimental conditions. We performed three types of ANOVAs on these data. In the first (initial target bearing), the analyzed variables were all manipulated as part of the experimental design. In the second (target updating), the data were categorized according to the participants' pointing responses. In the third (recalibrated experimental data), data from the control conditions were used to calibrate the raw data.

Analysis by initial target bearing. Figure 5 shows the average signed pointing error from the first ANOVA. The $0^{\circ}$ and $65^{\circ}$ targets were each sometimes paired with $125^{\circ}$ body rotations, but the maximum rotation paired with the $25^{\circ}$ target was $100^{\circ}$; we therefore omitted the trials involving $125^{\circ}$ rotations for this analysis, so that the range of body rotations combined with each target was $25^{\circ}-100^{\circ}$. Figure 5 shows all the data, including the unanalyzed conditions. The primary finding from this analysis is that, although all the groups tended to produce smaller pointing responses than required, there were reliable differences between groups $\left[F(2,18)=6.485, M S_{\mathrm{e}}=11,402, p=\right.$ .008 ]. The parietal group's average pointing error was $27^{\circ}$ in the experimental trials, whereas the healthy and brain-injured control groups averaged $-16^{\circ}$ and $-9^{\circ}$, respectively. Pairwise planned contrasts $(p=.05)$ showed that the only reliable differences were between the parietal group and the two control groups. There was neither 
Table 3

Source Tables for Analysis of Variance of Pointing Errors

\begin{tabular}{lrrr}
\hline \multicolumn{1}{c}{ Condition } & $F$ Ratio & $M S_{\mathrm{e}}$ & $p$ Value \\
\hline Point-to-origin & & & \\
$\quad$ Group $(2,18)$ & 5.069 & $1,839.31$ & .018 \\
$\quad$ Side $(1,18)$ & 0.202 & 15.73 & .659 \\
$\quad$ Side $\times$ group $(2,18)$ & 0.702 & 54.72 & .509 \\
No crossover & & & \\
$\quad$ Group $(2,18)$ & 8.899 & $1,874.02$ & .002 \\
Side $(1,18)$ & 0.442 & 39.48 & .514 \\
$\quad$ Side $\times$ group $(2,18)$ & 0.134 & 11.93 & .876 \\
Crossover & & & \\
$\quad$ Group $(2,18)$ & 2.873 & 503.38 & .083 \\
$\quad$ Side $(1,18)$ & 2.143 & 193.24 & .161 \\
$\quad$ Side $\times$ group $(2,18)$ & 0.230 & 20.74 & .797 \\
\hline
\end{tabular}

a main effect of rotation direction $[F(1,18)=0.822$, $\left.M S_{\mathrm{e}}=648.91, p=.376\right]$ nor a direction $\times$ group interaction $\left[F(2,18)=0.282, M S_{\mathrm{e}}=222.39, p=.758\right]$.

The pointing errors were also differentiated according to the initial target bearing $\left[F(2,36)=4.816, M S_{\mathrm{e}}=\right.$ $1,135.99, p=.014]$, but the three groups were not differently affected by this factor $\left[F(4,36)=1.494, M S_{\mathrm{e}}=\right.$ $116.59, p=.730]$. Performance was generally best in the context of the $65^{\circ}$ target, with an average signed error of $-14^{\circ}$, whereas error in the context of the other two targets was quite similar $\left(-19^{\circ}\right)$. The target effect depended on rotation magnitude $\left[F(6,108)=3.096, M S_{\mathrm{e}}=176.49, p=\right.$ .008 ], with pointing error tending to become even more negative with increases in rotation magnitude for the origin and $25^{\circ}$ targets, but to a lesser extent in the context of the $65^{\circ}$ target. A possible explanation for the target-bearing effect draws from the observation that the participants tended to turn their heads slightly when viewing the more eccentric targets and then return them to the straight ahead position before the rotation. This may have partially activated the semicircular canals prior to the whole-body rotation, effectively adding a small constant amount to the registered rotation.

Analysis by target updating in contralesional hemispace. The significant effect of initial target bearing dictated that we could not directly compare point-to-origin, crossover, and no-crossover trials, because they differed substantially in terms of the initial target bearing. Whereas point-to-origin trials exclusively involved the origin, crossover trials predominantly involved the $25^{\circ}$ targets, and no-crossover trials made use of the $25^{\circ}$ and $65^{\circ}$ targets in more equal measure. We therefore performed separate ANOVAs on the three conditions. In each test, the critical comparison for evaluating our hypothesis was the group $\times$ rotation direction (ipsi- vs. contralesional) interaction. The source tables for these analyses are presented in Table 3, and the data underlying them is summarized in Figure 6 . The point-to-origin and no-crossover trials both showed a main effect of group, with the parietal patients tending to undershoot more than the other two groups. More important for our hypotheses, however, there was no group $\times$ rotation direction interaction, for any of the three trial types.
Interestingly, the patient with the most severe neglect (L.D.) became quite disoriented on all the trials involving body rotations. Although he could point accurately in the no-rotation calibration trials, his pointing errors were large in the experimental trials (see asterisks in Figure 6). His errors in the no-crossover and point-to-origin trials were largest after rotations toward his contralesional and ipsilesional sides, respectively. Both of these rotations maintained the remembered target in his contralesional hemispace, which is consistent with our hypothesis that target updating impairments would be associated with the amount of updating in the contralesional hemispace, and not with the direction of body rotation. L.D.'s performance on the crossover trials, however, was not consistent with our predictions: On these trials, his largest errors occurred when the target crossed into his contralesional hemispace after a body rotation toward his ipsilesional side. This being the case, and taking into account L.D.'s unusually large pointing errors on rotation trials, we remain cautious regarding the possible support that L.D.'s case might lend to our hypotheses.

Recalibrated experimental data. In an attempt to increase the sensitivity of the analyses, we made use of data from the perceptuomotor performance and midline shift trials to transform the raw pointing errors. In one analysis, we used the least squares criterion to fit straight lines through the function relating the physical target direction with the pointing responses in the perceptuomotor performance trials. Our previous analyses showed that performance on the visually guided and no-vision perceptuomotor performance trials did not differ reliably, suggesting that the regression parameters of the perceptuomotor performance data could be used to estimate the combined contribution of visual localization, perceptuomotor calibration, and memory toward overall error. We accordingly used the parameters to transform the experimental data, thereby effectively removing these sources of error. In another analysis, we fit straight lines through the function relating stimulus rotations to straight ahead judgments on the midline shift trials. We used these parameters to estimate and then remove the midline shifts that would be obtained for each of the body rotations used on the experimental trials. We then applied the perceptuomotor transformation described above. Although these transformations did decrease overall error, the results differed in only minor ways from analyses on the untransformed data. Specifically, the analyses did not uncover any new effects, and there were again no group $\times$ rotation direction interactions.

\section{DISCUSSION}

Although the data analysis was complex, several clear findings emerged. All the participants tended to produce smaller responses than required when pointing to remembered target locations after whole-body rotations. This is consistent with the findings of other studies involving neurologically intact participants (Blouin et al., 

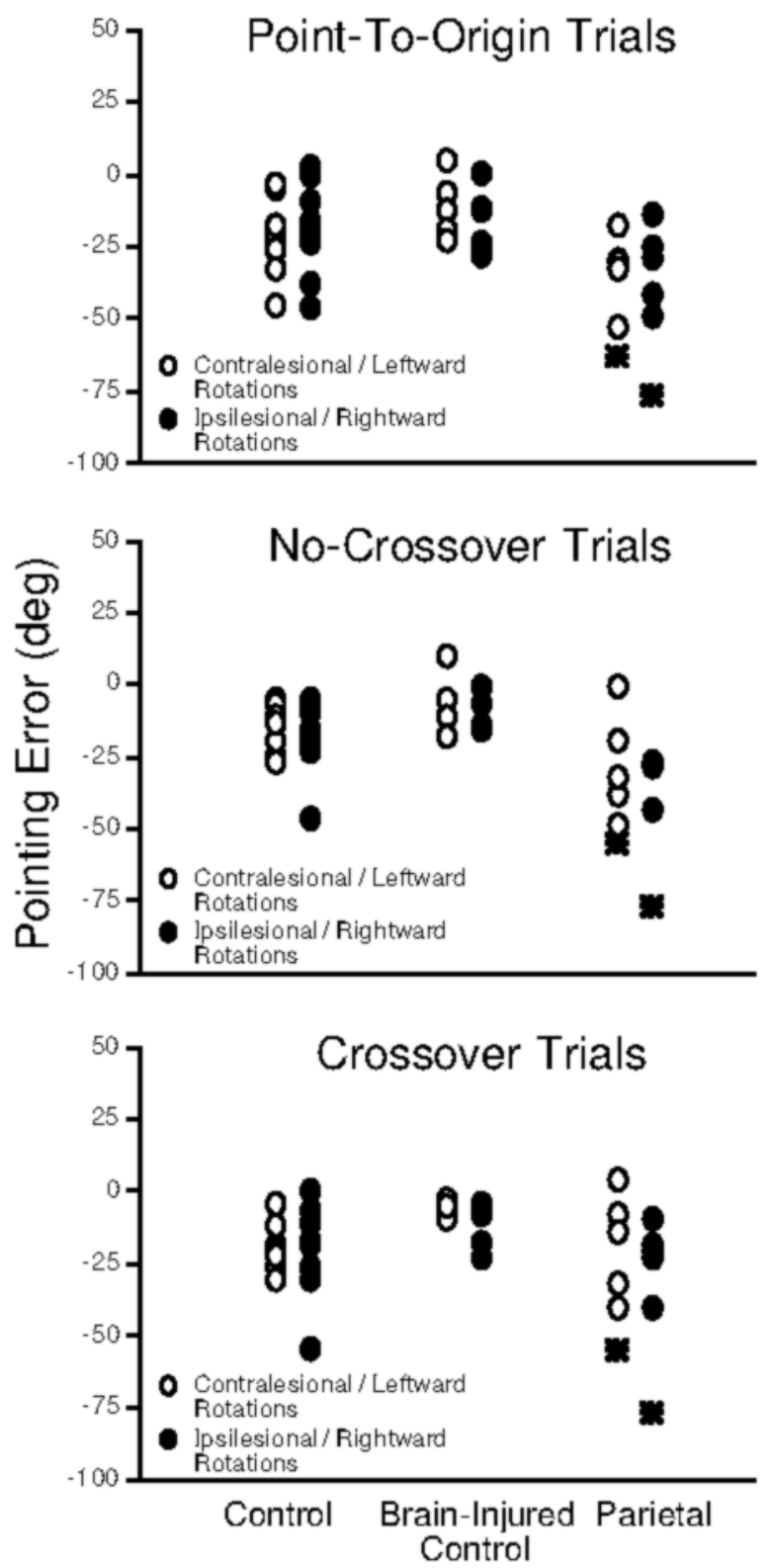

Figure 6. Mean signed pointing errors for the participants in the three groups. Each symbol represents the mean error for 1 individual. Open circles show data from trials involving contralesional rotations (leftward, for the healthy control group), and filled circles show data from trials involving ipsilesional rotations (rightward, for the healthy control group). Asterisks show the data for patient L.D. in the parietal group, who had the most severe hemispatial neglect. Negative values signify undershoots of the required value.

1995; Yardley, Gardner, Lavie, \& Gresty, 1999). The parietal group, however, performed significantly more poorly. Although deficits in controlling certain motor responses, such as eye movements, have been associated with parietal damage (Behrmann et al., 2002; Israël et al., 1995; Niemeier \& Karnath, 2000), our patients' manual point- ing responses were generally well calibrated when no rotations were involved. This suggests that the pointing deficits observed on trials involving body rotations are not due to impairments in perceiving the initial target location or controlling the pointing response. Two candidate sources of error that remain are deficits in sensing the body rotation or in updating the remembered target location. In previous studies that have found underestimations of body rotation in parietal patients (Farrell \& Robertson, 2000; Tropper et al., 1991), the possibility exists that the deficits were caused by encroachment of the lesion onto nearby vestibular cortical regions, rather than by parietal damage per se. In a study designed, in part, to distinguish between parietal and vestibular cortical contributions to rotational sensing, parietal damage was not associated with deficits in sensing rotations (Israël et al., 1995). We cannot rule out some vestibular cortical damage in our parietal group, so the general pattern of underestimation they exhibited may well be due to deficits in sensing body rotations caused by vestibular cortical lesions. The injuries did not eliminate the ability to perceive the rotations, although more extensive lesions may have that effect; instead, they apparently reduced the gain of the perceived body rotation, regardless of the direction of rotation.

Our primary interest, however, was in the degree to which parietal patients could update the location of remembered targets. The critical issue in this regard was not the overall pattern of underestimations but, rather, whether or not PPC-injured individuals would perform especially poorly when targets had to be updated in the contralesional region. Despite reasons to expect there to be more error in the contralesional region, our surprising finding was the PPC patients updated targets equally well on either side of their body midline once they had localized it. Even when PPC patients are impaired at detecting or responding to objects or events on one side of their body midline, spatial processing in that region is by no means obliterated. Our results confirm that target updating proceeds in this region, and indeed, even target detection can be improved in the contralesional region, given appropriate sensory or verbal cues (Riddoch \& Humphreys, 1983). Two classes of explanations for this effect have been proposed, with one arguing that such cuing alters the spatial allocation of attention, and the other arguing that cuing alters the representations of spatial locations (Vallar, Guariglia, \& Rusconi, 1997). Our results do not allow us to distinguish between these two alternatives, but it may be that updating the location of remembered targets engages processes similar to those underlying the amelioration of neglect symptoms by sensory or verbal cuing.

Even though the right PPC has been implicated in updating certain kinds of sensory signals (e.g., the eye position signal and, perhaps, arm position), it does not appear to be critically involved in updating during locomotion along linear or rotational trajectories. Patients with righthemisphere PPC lesions remain able to update their lo- 
cation while walking along linear paths (Philbeck et al., 2000), and there are no side-specific deficits in updating remembered target locations during whole-body rotations. Although the PPC may yet play a role by way of monitoring the vestibular signal, the weight of existing evidences argues against that possibility (Israël et al., 1995). There are several remaining possibilities for PPC involvement in these tasks. One is that adequate performance may be maintained by the contralesional PPC regardless of which hemisphere has been damaged. The left-hemisphere PPC might also play a more dominant role in target updating than the right. Our single left-hemisphere PPC patient's performance was unremarkable within the PPC group, arguing against such a left-hemisphere dominance, but more research would be needed to resolve this issue. Another possibility is that the superior PPC is more heavily involved than the inferior region. Superior PPC damage in humans is associated with deficits in transforming visually specified representations of target locations into appropriate motor coordinates for the control of action (Perenin \& Vighetto, 1988); the superior PPC might play a similar role in generating a pointing response, using our paradigm, despite the short nonvisual delay before responses were executed. Neglect, meanwhile, is more often associated with damage to the inferior PPC and the superior temporal lobe (Bisiach \& Vallar, 2001; Karnath, Ferber, \& Himmelbach, 2001).

Our results contrast with those obtained using the double-step saccade paradigm, in which PPC-injured patients were impaired at updating the eye position signal (Duhamel et al., 1992; Heide et al., 1995). How can one account for this difference? It is possible that there are response-specific dissociations in spatial updating, so that an individual who shows impairments in the doublestep saccade paradigm may not be impaired in our targetupdating task. This is an intriguing possibility, but as yet no PPC-injured patients have been tested on both tasks to investigate this issue. Response-specific dissociations could shed light on the reference frames underlying target updating. One aspect of our method is that multiple frames of reference were available for encoding the initial target location. In addition to the possibility of allocentric (environment-referenced) encoding, the targets could be separately encoded in egocentric coordinates relative to the trunk, head, or eyes. The multiplicity of these available reference frames may have increased the robustness of the updating processes involved in our task, perhaps drawing from representations in intact brain areas that continued to function normally.

Previous studies involving whole-body rotations have tended to use rapid responses, such as saccadic eye movements (Israël et al., 1995; Tropper et al., 1991) or a decisive pointing motion (Farrell \& Robertson, 2000). There is some indication that rapid responses of this nature may be controlled by a relatively short-lived spatial representation that is specialized for the visual control of action within an egocentric frame of reference (Day \& Lyon,
2000; Desmurget et al., 1999; Milner \& Goodale, 1995; Pisella et al., 2000; see also Aguirre \& D'Esposito, 1999, and Farrell, 1996). In this view, a more long-lived representation, presumably subserved by cortical regions lying outside the PPC, controls nonballistic or delayed responses. Thus, there is some indication that the type of response required can determine what spatial representation (and therefore, what anatomical substrate) ultimately controls the response. Consistent with this view, the PPC patients studied by Israël et al. (1995) were deficient only in controlling the first saccade on a trial, suggesting that the first saccade may have been controlled by the lesioned PPC; subsequent corrective saccades (presumably driven by a less distorted spatial representation) brought the eye to a final position that was not significantly different from those produced by healthy control participants.

The foregoing evidence suggests a possible explanation for why we did not observe updating deficits on the contralesional side of the body midline. Our manual aiming responses not only were delayed (by virtue of the passive body rotation), but also were performed without visual control. These aspects suggest that the manual aiming response may be controlled by a spatial representation that is subserved by undamaged brain tissue outside the PPC. If such is the case, one would not expect PPC lesions to impair updating of contralesional targets as indicated by the manual aiming response.

\section{REFERENCES}

Aguirre, G. K., \& D’Esposito, M. (1999). Topographical disorientation: A synthesis and taxonomy. Brain, 122, 1613-1628.

Andersen, R. A., Snyder, L. H., Bradley, D. C., \& Xing, J. (1997). Multimodal representation of space in the posterior parietal cortex and its use in planning movements. Annual Review of Neuroscience, 20, 303-330.

Behrmann, M., Ghiselli-Crippa, T., \& Di Matteo, I. (2002). Impaired initiation but not execution of eye movements in patients with hemispatial neglect. Behavioral Neurology, 13, 1-16.

Behrmann, M., Nelson, J., \& Sekuler, E. (1998). Visual complexity in letter-by-letter reading: "Pure" alexia is not so pure. Neuropsychologia, 36, 1115-1132.

Bisiach, E., Pattini, P., Rusconi, M. L., Ricci, R., \& Bernardini, B. (1997). Unilateral neglect and space constancy during passive locomotion. Cortex, 33, 313-322.

Bisiach, E., \& VAllar, G. (2001). Unilateral neglect in humans. In F. Boller \& J. Grafman (Eds.), Handbook of neuropsychology (2nd ed., Vol. 1, pp. 459-502). Amsterdam: North-Holland.

Blouin, J., Gauthier, G. M., \& Vercher, J.-L. (1995). Failure to update the egocentric representation of the visual space through labyrinthine signal. Brain \& Cognition, 29, 1-22.

Bonda, E., Petrides, M., Frey, S., \& Evans, A. (1995). Neural correlates of mental transformations of the body-in-space. Proceedings of the National Academy of Sciences, 92, 11180-11184.

Colby, C. L., Duhamel, J. R., \& Goldberg, M. E. (1996). Visual, presaccadic, and cognitive activation of single neurons in monkey lateral intraparietal area. Journal of Neurophysiology, 76, 2841-2852.

Colby, C. L., \& Goldberg, M. E. (1999). Space and attention in parietal cortex. Annual Review of Neuroscience, 22, 319-349.

DAY, B. L., \& LYON, I. N. (2000). Voluntary modification of automatic arm movements evoked by motion of a visual target. Experimental Brain Research, 130, 159-168. 
Desmurget, M., Epstein, C. M., Turner, R. S., Prablanc, C., AlexanDER, G. E., \& GRAFTon, S. T. (1999). Role of the posterior parietal cortex in updating reaching movements to a visual target. Nature Neuroscience, 2, 563-567.

DeSouza, J. F. X., Dukelow, S. P., Gati, J. S., Manon, R. S., AnderSEN, R. A., \& VILIS, T. (2000). Eye position signal modulates a human parietal pointing region during memory guided movements. Journal of Neuroscience, 20, 5835-5840.

Duhamel, J. R., Goldberg, M. E., Fitzgibbon, E. J., Sirigu, A., \& Grafman, J. (1992). Saccadic dysmetria in a patient with a right frontoparietal lesion: The importance of corollary discharge for accurate spatial behaviour. Brain, 115, 1387-1402.

FARrell, M. J. (1996). Topographical disorientation. Neurocase, 2, 509-520.

FARrell, M. J., \& Robertson, I. H. (2000). The automatic updating of egocentric spatial relationships and its impairment due to right posterior cortical lesions. Neuropsychologia, 38, 585-595.

Friberg, L., Olsen, T. S., Roland, P. E., Paulson, O. B., \& Lassen, N. A. (1985). Focal increase of blood flow in the cerebral cortex of man during vestibular stimulation. Brain, 108, 609-623.

Heide, W., Blankenburg, M., Zimmermann, E., \& Kompf, D. (1995). Cortical control of double-step saccades: Implications for spatial orientation. Annals of Neurology, 38, 739-748.

Israël, I., Rivaud, S., Gaymard, B., Berthoz, A., \& PierrotDeseilligny, C. (1995). Cortical control of vestibular-guided saccades in man. Brain, 118, 1169-1183.

KarNath, H.-O. (1994). Subjective body orientation in neglect and the interactive contribution of neck muscle proprioception and vestibular stimulation. Brain, 117, 1001-1012.

Karnath, H.-O., \& Ferber, S. (1999). Is space representation distorted in neglect? Neuropsychologia, 37, 7-15.

Karnath, H.-O., Ferber, S., \& Himmelbach, M. (2001). Spatial awareness is a function of the temporal not the posterior parietal lobe. Nature, 411, 951-953.

Karnath, H.-O., \& Perenin,M.-T. (1998). Tactile exploration of peripersonal space in patients with neglect. NeuroReport, 9, 2273-2277.

Lobel, E., Kleine,J. F., Bihan, D. L., Leroy-Willig, A., \& Berthoz, A. (1998). Functional MRI of galvanic vestibular stimulation. Journal of Neurophysiology, 80, 2699-2709.

Loomis, J. M., Klatzky, R. L., Golledge, R. G., \& Philbeck, J. W. (1999). Human navigation by path integration. In R. G. Golledge (Ed.), Wayfinding behavior: Cognitive mapping and other spatial processes (pp. 125-151). Baltimore: Johns Hopkins University Press.

Mapelli, D., \& Behrmann, M. (1997). The role of color in object recognition: Evidence from visual agnosia. Neurocase, 3, 237-247.

Milner, A. D., \& Goodale, M. A. (1995). The visual brain in action. Oxford: Oxford University Press.

Niemeier, M., \& Karnath, H.-O. (2000). Exploratory saccades show no direction-specific deficit in neglect. Neurology, 54, 515-518.

Perenin, M.-T., \& Vighetto, A. (1988). Optic ataxia: A specific disruption in visuomotor mechanisms: I. Different aspects of the deficit in reaching for objects. Brain, 111, 643-674.

Petit, L., Orssaud, C., Tzourio, N., Mazoyer, B., \& Berthoz, A. (1997). Superior parietal lobule involvement in the representation of visual space: A PET review. In P. Thier \& H.-O. Karnath (Eds.), Parietal lobe contributions to orientation in $3 D$ space (pp. 77-91). Heidelberg: Springer-Verlag.

Philbeck, J. W., Behrmann, M., Black, S. E., \& Ebert, P. (2000). Intact spatial updating during locomotion after right posterior parietal lesions. Neuropsychologia, 38, 950-963.

Pisella, L., Grea, H., Tilikete, C., Vighetto, A., Desmurget, M., Rode, G., Boisson, D., \& Rossetti, Y. (2000). An "automatic pilot" for the hand in human posterior parietal cortex: Toward reinterpreting optic ataxia. Nature Neuroscience, 3, 729-736.

Pizzamiglio, L., Iaria, G., Berthoz, A., Galati, G., \& Guariglia, C. (in press). Navigation in neglect patients. In H.-O. Karnath, A. D. Milner, \& G. Vallar (Eds.), The cognitive and neural bases of neglect. Oxford: Oxford University Press.

PoucET, B. (1993). Spatial cognitive maps in animals: New hypotheses on their structure and neural mechanisms. Psychological Review, 100, 163-182.

Riddoch, M. J., \& Humphreys, G. W. (1983). The effect of cueing on unilateral neglect. Neuropsychologia, 21, 589-599.

RIESER, J. J. (1989). Access to knowledge of spatial structure at novel points of observation. Journal of Experimental Psychology: Learning, Memory, \& Cognition, 15, 1157-1165.

Rode, G., Charles, N., Perenin, M. T., Vighetto, A., Trillet, M., \& AImARD, G. (1992). Partial remission of hemiplegia and somatoparaphrenia through vestibular stimulation in a case of unilateral neglect. Cortex, 28, 203-208.

Rode, G., \& Perenin, M. T. (1994). Temporary remission of representational hemineglect through vestibular stimulation. NeuroReport, $\mathbf{5}$, 869-872.

Sirigu, A., Duhamel, J.-R., Cohen, L., Pillon, B., Dubois, B., \& AgID, Y. (1996). The mental representation of hand movements after parietal cortex damage. Science, 273, 1564-1568.

Snyder, L. H. Batista, A. P., \& ANdersen, R. A. (1997). Coding of intention in the posterior parietal cortex. Nature, 386, 167-170.

STEIN, J. F. (1992). The representation of egocentric space in posterior parietal cortex. Behavioral \& Brain Sciences, 15, 691-700.

Tropper, J., Jones, G. M., Bloomberg, J., \& Fadlallah, H. (1991). Vestibular perceptual deficits in patients with parietal lobe lesions: A preliminary study. Acta Oto-Laryngologica, Suppl. 481, 528-533.

Uemura, T., Arai, Y., \& Shimazaki, C. (1980). Eye-head coordination during lateral gaze in normal subjects. Acta Oto-Laryngologica, 90, 191-198.

VALlar, G. (1998). Spatial hemineglect in humans. Trends in Cognitive Sciences, 2, 87-96.

Vallar, G., Guariglia, C., \& Rusconi, M. L. (1997). Modulation of the neglect syndrome by sensory stimulation. In P. Thier \& H.-O. Karnath (Eds.), Parietal lobe contributions to orientation in $3 D$ space (pp. 555-578). Heidelberg: Springer-Verlag.

Vecera, S. P., \& Behrmann, M. (1997). Spatial attention does not require preattentive grouping. Neuropsychology, 11, 30-43.

Vitte, E., Derosier, C., Caritu, Y., Berthoz, A., Hasboun, D., \& Soulie, D. (1996). Activation of the hippocampal formation by vestibular stimulation: A functional magnetic resonance imaging study. Experimental Brain Research, 112, 523-526.

Wilson, B., Cockburn, J., \& Halligan, P. (1987). Behavioral inattention test. Bury St. Edmunds, U.K.: Thames Valley Test Company.

Wolpert, D. M., \& Ghahramani, Z (2000). Computational principles of movement neuroscience. Nature Neuroscience, 3 (Suppl.), 12121217.

Wolpert, D. M., Goodbody, S. J., \& Husain, M. (1998). Maintaining internal representations: The role of the human superior parietal lobe. Nature Neuroscience, 1, 529-533.

Yardley, L., Gardner, M., Lavie, N., \& Gresty, M. (1999). Attentional demands of perception of passive self-motion in darkness. Neuropsychologia, 37, 1293-1301.

Zacks, J., Ry pma, B., Gabrieli, J. D. E., Tversky, B., \& Glover, G. H. (1999). Imagined transformations of bodies: An fMRI investigation. Neuropsychologia, 37, 1029-1040.

\section{NOTES}

1. The healthy control and parietal groups were approximately age matched, whereas the brain-injured control group was somewhat younger on average. There was, however, substantial overlap in age between this group and the parietal group, and the age differences that do exist do not preclude studying these patients to evaluate the effect of brain injuries lying outside the PPC.

2 . After the study had begun, a second version of the table was constructed that could be dismantled and reassembled when needed to maximize laboratory usage; the table had a very similar appearance, apart from being lighter in color. The original design called for targets at $25^{\circ}$ and $75^{\circ}$, but unfortunately the $75^{\circ}$ targets on the second table were inadvertently positioned at $65^{\circ}$. The majority of the participants therefore saw targets at $25^{\circ}$ and $65^{\circ}$, but a subset (4 in the healthy control group, 
2 in the parietal group, and 1 in the brain-injured control group) saw targets at $25^{\circ}$ and $75^{\circ}$. The participants in all three groups pointed quite accurately in the perceptuomotor calibration trials, regardless of target position, so the impact of this difference is likely to be minor. We dealt with this issue when analyzing the data by calculating pointing error relative to the $65^{\circ}$ or $75^{\circ}$ targets (as appropriate), instead of using the raw data. In analyses that included target position as a variable, we grouped the data from the $65^{\circ}$ and $75^{\circ}$ target positions together. For simplicity, we will hereafter refer to the $65^{\circ}$ and $75^{\circ}$ target conditions collectively as $65^{\circ}$ target conditions. Note that the recalibrated experimental data analysis provided some means of taking the actual target positions into account.

(Manuscript received March 30, 2001; revision accepted for publication October 31, 2001.) 